九州大学学術情報リポジトリ

Kyushu University Institutional Repository

\title{
A FUZZY PERCEPTIVE VALUE FOR MULTI-VARIATE STOPPING PROBLEM WITH A MONOTONE RULE
}

Kurano, Masami

Faculty of Education, Chiba University

Yasuda, Masami

Faculty of Science, Chiba University

Nakagami, Jun-ichi

Faculty of Science, Chiba University

Yoshida, Yuji

Faculty of Economics and Business Administration, The University of Kitakyushu

https://doi.org/10.5109/16770

出版情報: Bulletin of informatics and cybernetics. 39, pp.1-9, 2007-12. Research Association of Statistical Sciences

バージョン :

権利関係 : 


\title{
A FUZZY PERCEPTIVE VALUE FOR MULTI-VARIATE STOPPING PROBLEM WITH A MONOTONE RULE
}

\author{
by \\ Masami Kurano, Masami YASUdA, Jun-ichi NAKAgAMI \\ and \\ Yuji YosHIDA
}

Reprinted from the Bulletin of Informatics and Cybernetics

Research Association of Statistical Sciences, Vol.39

FUKUOKA, JAPAN

2007 


\title{
A FUZZY PERCEPTIVE VALUE FOR MULTI-VARIATE STOPPING PROBLEM WITH A MONOTONE RULE
}

\author{
By \\ Masami Kurano, Masami Yasuda ${ }^{\dagger} \quad$ Jun-ichi NAKAgAMI ${ }^{\ddagger}$ \\ and \\ Yuji YosHIDA ${ }^{\S}$
}

\begin{abstract}
This paper discusses a perception-based theory for a multi-variate stopping problem with a monotone rule by Yasuda et al. (1982). The problem is developed by using the perceptive analysis of the previous work(Kurano et al. (2004)). We will show a recursive equation which determines the perceptive value of its equilibrium point for the model.
\end{abstract}

Key Words and Phrases: Equilibrium point, Fuzzy perception equilibrium payoff, Fuzzy perception stopping problem, Monotone logical rule, Multi-variate stopping problem.

\section{Introduction}

In a social life or in business, a group decision-making cannot be avoided and be carried up by taking each individual opinions into the whole group decision. In our previous works( Yasuda et al. (1982)), the multi-variate stopping problem by $p$-players $(p \geq 2)$ has been formulated. The key point of a group decision at each stage is to sum up each individual declaration(opinion) by cooperating a monotone logical rule in order to make the group decision, that is, "stop" or "continue" the multi-variate stochastic process.

In order to review the result and extend it to our fuzzy model, we firstly consider the following processes: Let $X=\left(X_{1}, \cdots, X_{N}\right)$ be a sequence of $p$-dimensional random vectors on a probability space $(\Omega, \mathscr{B}, P)$. The planning time horizon is $N$ and $p$ means a number of players. That is, $X_{n}=\left(X_{n}^{1}, \cdots, X_{n}^{p}\right), n=1, \cdots, N$ with $X_{n}^{i}(i=1, \cdots, p)$ a payoff for player $i$ when the process have stopped at stage $n$.

To describe an individual declaration of each player, let us consider $\{0,1\}$-valued, $\left\{X_{n}\right\}$ adapted process $d^{i}=\left(d_{1}^{i}, \cdots, d_{N}^{i}\right), i=1, \cdots, p$. Such a sequence $d^{i}$, called an individual strategy for player $i$, is interpreted as follows: On the basis of the observed value $X_{1}, \cdots, X_{n}$ at stage $n$, the decision $d_{n}^{i}=1$ means that player $i$ declares to stop the process and $d_{n}^{i}=0$ is otherwise. The set of all individual strategies of player $i$ will be denoted by $D^{i}(X)$ depending on $X=\left\{X_{n}^{i}, n=\right.$ $1, \cdots, N, i=1, \cdots, p\}$

A $\{0,1\}$-valued $p$-variate logical function $\pi=\pi\left(x^{1}, \cdots, x^{p}\right):\{0,1\}^{p} \rightarrow\{0,1\}$ is said to be monotone if $\pi(0, \cdots, 0)=0, \pi(1, \cdots, 1)=1$ and $\pi\left(x^{1}, \cdots, x^{p}\right) \leq \pi\left(y^{1}, \cdots, y^{p}\right)$ whenever $x^{i} \leq y^{i}$ for each $i$ (Fishburn (1971), Yasuda et al. (1982)). A stopping time of the process is defined by

\footnotetext{
* Faculty of Education, Chiba University, Chiba 263-8522 Japan,

$\dagger$ Faculty of Science, Chiba University, Chiba 263-8522 Japan,

¥ Faculty of Science, Chiba University, Chiba 263-8522 Japan,

$\S$ Faculty of Economics and Business Administration, The University of Kitakyushu, Kitakyushu 802-8577 Japan,
} 
the individual strategy and the monotone rule: For any $d=\left(d^{1}, \cdots, d^{p}\right) \in D(X):=\prod_{i=1}^{p} D^{i}(X)$ with $d^{i}=\left(d_{1}^{i}, \cdots, d_{N}^{i}\right) \in D^{i}(X), i=1, \cdots, p$,

$$
t_{\pi}(d) \triangleq\left\{\text { smallest } n \text { such that } \pi\left(d_{n}^{1}, \cdots, d_{n}^{p}\right)=1\right\}
$$

with $d=\left(d^{i} ; i=1, \cdots, p\right), d^{i}=\left(d_{n}^{i} ; n=1, \cdots, N\right)$. Thus, the expected payoff of player $i$ for $X=\left\{X_{n}^{i}, n=1, \cdots, N, i=1, \cdots, p\right\}$ with the individual strategy $d$ under the monotone rule $\pi$ is defined by $E\left[X_{t_{\pi}(d)}^{i}\right]$.

Under the assumption that the value of random vectors is known to all players, an equilibrium point ${ }^{*} d=\left({ }^{*} d^{1}, \cdots,{ }^{*} d^{p}\right) \in D$ is explicitly given and its corresponding equilibrium payoff is calculated by a recursive equation of Dynamic Programming in Yasuda et al. (1982). Hereafter the above problem will be denoted by $\operatorname{PROB}\{X \mid \pi, N\}$ to distinguish it from a perceptive model given in the sequel section. In many practical situation, we are often forced with the case that the value of the sequence of random variable is partially observed by dimness of perception or measurement imprecision. In order to treat with such a situation, we use a fuzzy perception function(Baswell/Taylor (1987)) by which the perception level of each random variable is represented.

In this paper, we give a fuzzy perception model F-PROB $\{\widetilde{X} \mid \pi, N\}$ which extends a probability model $\operatorname{PROB}\{X \mid \pi, N\}$ and characterize the perception value of each player's equilibrium payoff. The payoff can be calculated by a fuzzy recursive equation. This problem is motivated by Zadeh's paper(Zadeh (2002)) on a perceptive-based probability theory in which the perception value of the objective function under possibility constraints is introduced and calculated using a generalized extension principle.

The perceptive analysis developed in this paper is related to our previous works. A model of stopping problems is formulated in Kurano et al. (2004) and that of Markov decision processes is in Kurano et al. (2005a) and Kurano et al. (2005b). However the basic assumption implemented in the previous stopping problem is different from this paper. The model which we want to consider here is restricted within two decision case, that is, $\{0,1\}$. Its general formulation of a fuzzy decision case seems to be a quite difficult.

\section{A fuzzy perception function and a pseudo order}

In this section we will give some notion and the definition of fuzzy perception functions. Also, a pseudo order on the set of fuzzy sets is described because the fuzzy perception model for multi-variate stopping needs this notion.

For any set $A$, a fuzzy set on $A$ will be denoted by its membership function $\widetilde{a}: A \rightarrow$ $[0,1]$. The $\alpha$-cut of $\widetilde{a}$ is given by $\widetilde{a}_{\alpha}:=\{x \in A \mid \widetilde{a}(x) \geq \alpha\}(\alpha \neq 0)$ and, for $\alpha=0, \widetilde{a}_{0}:=$ $\operatorname{cl}\{x \in A \mid \widetilde{a}(x)>0\}$ where cl means the closure of set. For other notions of fuzzy sets, refer to Dubois/Prade (1980), Zadeh (1965). Let $\mathbb{R}$ be the set of all real numbers and $\mathscr{F}(\mathbb{R})$ the set of all fuzzy sets on $\mathbb{R}$, and $\widetilde{\mathbb{R}} \subset \mathscr{F}(\mathbb{R})$ the set of all fuzzy numbers on $\mathbb{R}$, i.e., $\widetilde{r} \in \widetilde{\mathbb{R}}$ means that $\widetilde{r}: \widetilde{\mathbb{R}} \rightarrow[0,1]$ is normal, upper semi-continuous and fuzzy convex and has a compact support. Let $\mathbb{C}$ be the set of all bounded and closed intervals of $\mathbb{R}$. Then, for any $\widetilde{r} \in \widetilde{\mathbb{R}}$, it obviously holds that $\widetilde{r}_{\alpha} \in \mathbb{C}(\alpha \in[0,1])$. So, we write $\widetilde{r}_{\alpha}=\left[{ }^{-} r_{\alpha},{ }^{+} r_{\alpha}\right](\alpha \in[0,1])$ as a closed interval. A map $\widetilde{X}: \Omega \rightarrow \widetilde{\mathbb{R}}$ is called a fuzzy perception function(Baswell/Taylor (1987)) or a fuzzy random variable (Puri/Ralescu (1986)) if, for each $\alpha$ and $\omega$, the closed interval $\widetilde{X}_{\alpha}(\omega)=\left[{ }^{-} \widetilde{X}_{\alpha}(\omega),{ }^{+} \widetilde{X}_{\alpha}(\omega)\right]$ is endowed with each measurable end points. 
Let denote all of integrable random variable on $(\Omega, \mathscr{B}, P)$ by $\mathscr{X}$. For any fuzzy random perception function $\widetilde{X}$, its expectation $E \widetilde{X} \in \widetilde{\mathbb{R}}$ is defined by

$$
E \widetilde{X}(x) \triangleq \sup \{\widetilde{\mu}(\widetilde{X})(X) ; X \in \mathscr{X}, E(X)=x\}
$$

provided that $\widetilde{\mu}(\widetilde{X})$ is a fuzzy set on $\mathscr{X}$ and $\widetilde{\mu}(\widetilde{X})(X)=\inf _{\omega \in \Omega} \widetilde{X}(\omega)(X(\omega))$ for all $X \in \mathscr{X}$. Obviously, we have

$$
E\left(\widetilde{X}_{\alpha}\right)=\left[\int-\widetilde{X}_{\alpha}(\omega) d P(\omega), \int+\widetilde{X}_{\alpha}(\omega) d P(\omega)\right]
$$

Next we define a binary relation $\preccurlyeq$ on $\mathscr{F}(\mathbb{R})$ as follows.

Definition 2.1 For any $\widetilde{s}, \widetilde{r} \in \mathscr{F}(\mathbb{R})$, a relation $\widetilde{s} \preccurlyeq \widetilde{r}$ holds if both of (i) and (ii) are satisfied simultaneously:

(i) For any $x \in \mathbb{R}$ and $\varepsilon>0$, there exists $y \in \mathbb{R}$ such that $x \leq y$ and $\widetilde{s}(x)<\widetilde{r}(y)+\varepsilon$.

(ii) For any $y \in \mathbb{R}$ and $\varepsilon>0$, there exists $x \in \mathbb{R}$ such that $x \leq y$ and $\widetilde{s}(x)>\widetilde{r}(y)-\varepsilon$.

This binary relation is an extension of the well known partial order called the fuzzy max order(Ramik/Rimanek (1985)). We should prove its property of pseudo ordering.

Lemma 2.1 The binary relation $\preccurlyeq$ is a pseudo order on $\mathscr{F}(\mathbb{R})$.

proof. First it trivially holds that $\widetilde{s} \preccurlyeq \widetilde{s}$ for $\widetilde{s} \in \mathscr{F}(\mathbb{R})$. The next is to show the transitivity. Assume that $\widetilde{s} \preccurlyeq \widetilde{t}$ and $\widetilde{t} \preccurlyeq \widetilde{r}$ for $\widetilde{s}, \widetilde{t}, \widetilde{r} \in \mathscr{F}(\mathbb{R})$. From $\widetilde{s} \preccurlyeq \widetilde{t}$ it follows that for any $x \in \mathbb{R}$ and $\varepsilon_{1}>0$, there exists $y \in \mathbb{R}$ which satisfy $x \leq y$ and $\widetilde{s}(x)<\widetilde{t}(y)+\varepsilon_{1}$. For this $y$, we select $z \in \mathbb{R}$ and $\varepsilon_{2}>0$ with $\widetilde{t}(y)<\widetilde{r}(z)+\varepsilon_{2}$ and $y \leq z$ since $\widetilde{t} \preccurlyeq \widetilde{r}$. Thus, we have $\widetilde{s}(s)<\widetilde{r}(z)+\varepsilon_{1}+\varepsilon_{2}$ with $x \leq z$. Similarly, there exists $x \in \mathbb{R}$ such that $\widetilde{s}(x)>\widetilde{r}(z)-\varepsilon$ and $x \leq z$ for any $z \in \mathbb{R}$ and $\varepsilon>0$. These shows that $\widetilde{s} \preccurlyeq \widetilde{r}$, which completes the proof.

\section{Fuzzy perception model of multi-variate stopping}

In this section a fuzzy perception model for multi-variate stopping problem is defined. Before the definition, we need a next assumption on the given probability space which is important to show the continuity of random vectors. This cause the determination of each individual decision.

Assumption 3.1 The probability measure $P$ with underling space $(\Omega, \mathscr{B}, P)$ is assumed to be non atomic.

Let denote by $\mathscr{X}^{p \times N}$ the set of all $N$-dimensional row random vector whose elements are mutually independent $p$-dimensional column random vectors. That is, a random variable $X_{n}^{i} \in \mathscr{X}$ means a payoff for players $i=1, \cdots, p$ at time-parameter $n=1, \cdots, N$. Composing each of time stage and whole of players payoff, it is constructed as

$$
\mathscr{X}^{p \times N} \triangleq\left\{\begin{array}{l|l}
X=\left(X_{1}, \cdots, X_{N}\right) & \begin{array}{c}
X_{n}=\left(X_{n}^{1}, \cdots, X_{n}^{p}\right)^{T} \in \mathscr{X}^{p} \text { and } X_{n}^{i} \in \mathscr{X} \\
\text { are mutually independent for } n=1, \cdots, N, \\
i=1, \cdots, p
\end{array}
\end{array}\right\}
$$

where $(\cdots)^{T}$ is the transpose of a vector. 
Definition 3.1 An individual stopping strategy function $\delta^{i}$ of player $i(i=1, \cdots, p)$ are defined as the map $\delta^{i}=\left(\delta_{n}^{i} ; n=1, \cdots, N\right): X \in \mathscr{X}^{p \times N} \rightarrow D^{i}(X)$.

We denote by $\Delta^{i}$ the set of all individual stopping strategy functions of player $i$ and $\Delta=$ $\Pi_{i=1}^{p} \Delta^{i}$. Simply call $\delta \in \Delta$ as a stopping strategy function. Let $\widetilde{X}=\left(\widetilde{X_{n}} ; n=1, \cdots, N\right)$ be a sequence of $p$-dimensional fuzzy perception function with $\widetilde{X_{n}}=\left(\widetilde{X}_{n}^{i}\right)$. For any $\delta=\left(\delta^{i} ; i=\right.$ $1, \cdots, p) \in \Delta$ and a monotone rule $\pi$, the $\delta$-stopped fuzzy perception payoff of player $i(i=$ $1, \cdots, p)$ is a fuzzy set on $\mathbb{R}$ and defined by

$$
\widetilde{v}^{i}(\delta)(x) \triangleq \sup _{X \in A} \mu_{\widetilde{X}}(X)
$$

where the supremum $X \in A$ is taken all over on the range of set

$$
A \triangleq\left\{X \in \mathscr{X}^{p \times N} \mid E\left(X_{t_{\pi}(\delta(X))}^{i}\right)=x\right\}
$$

and

$$
\mu_{\widetilde{X}}(X) \triangleq \inf _{\omega \in \Omega} \widetilde{X}_{1}\left(X_{1}(\omega)\right) \wedge \cdots \wedge \widetilde{X}_{N}\left(X_{N}(\omega)\right)
$$

and $\widetilde{X}_{n}\left(X_{n}(\omega)\right) \triangleq \widetilde{X}_{n}^{1}\left(X_{n}^{1}(\omega)\right) \wedge \cdots \wedge \widetilde{X}_{n}^{p}\left(X_{n}^{p}(\omega)\right)$.

The stopping strategy function ${ }^{*} \delta=\left({ }^{*} \delta^{i} ; i=1, \cdots, p\right) \in \Delta$ is called an equilibrium point (cd. Vorobe'v (1977)) if for each $i(i=1, \cdots, p)$ it holds that

$$
\left.\widetilde{v}^{i}\left({ }^{*} \delta\right) \succcurlyeq \widetilde{v}^{i}{ }^{*} \delta^{-i} / \delta^{i}\right)
$$

for all $\delta^{i} \in \Delta^{i}$, where ${ }^{*} \delta^{-i} / \delta^{i} \triangleq\left({ }^{*} \delta^{1}, \cdots,{ }^{*} \delta^{i-1}, \delta^{i},{ }^{*} \delta^{i+1}, \cdots,{ }^{*} \delta^{p}\right)$.

The objective of our fuzzy perception problem is to find an equilibrium point ${ }^{*} \delta \in \Delta$ and to calculate the equilibrium fuzzy perception payoff $\widetilde{v}^{i}\left({ }^{*} \delta\right)(i=1, \cdots, p)$. This problem will be denoted by F-PROB $\{\widetilde{X} \mid \pi, N\}$ in the afterwards.

\section{Equilibrium fuzzy perception payoffs}

In order to discuss F-PROB $\{\widetilde{X} \mid \pi, N\}$, we need the following results on $\operatorname{PROB}\{X \mid \pi, N\}$ given in the previous work(Yasuda et al. (1982)).

For any $p$-dimensional random variables $\left\{X_{n}=\left(X_{n}^{i} ; i=1, \cdots, p\right) ; n=1, \cdots, N\right\}$, we define a sequence of vectors $\left\{v_{n}=\left(v_{n}^{i} ; i=1, \cdots, p\right) ; n=1, \cdots, N\right\}$ inductively by, for each $i=1, \cdots, p$,

$$
\left\{\begin{aligned}
v_{1}^{i} & =E\left(X_{N}^{i}\right) \\
v_{n+1}^{i} & =v_{n}^{i}+\alpha^{i}\left(v_{n} \mid X_{N-n}\right) E\left(X_{N-n}^{i}-v^{i}\right)^{+}-\beta^{i}\left(v_{n} \mid X_{N-n}\right) E\left(X_{N-n}^{i}-v^{i}\right)^{-}
\end{aligned}\right.
$$

where

$$
\begin{aligned}
& \alpha^{i}\left(v_{n} \mid X_{N-n}\right)=P\left[\pi\left(I_{C_{n}^{1}}, \cdots, I_{C_{n}^{i-1}}, 1, I_{C_{n}^{i+1}}, \cdots, I_{C_{n}^{p}}\right)=1\right], \\
& \beta^{i}\left(v_{n} \mid X_{N-n}\right)=P\left[\pi\left(I_{C_{n}^{1}}, \cdots, I_{C_{n}^{i-1}}, 0, I_{C_{n}^{i+1}}, \cdots, I_{C_{n}^{p}}\right)=1\right]
\end{aligned}
$$

and $C_{n}^{j}=\left\{X_{N-n}^{j} \geq v_{n}^{j}\right\}(j=1, \cdots, p)$ and $I_{A}$ is an indicator of set $A$.

Lemma 4.1 (Corollary 2.2 in Yasuda et al. (1982)) For a sequence $\left\{v_{n}\right\}$ given in (4.1), define a strategy ${ }^{*} d=\left({ }^{*} d^{i}\right)$ with ${ }^{*} d^{i}=\left({ }^{*} d_{n}^{i}\right) \in D^{i}(X)$ by ${ }^{*} d_{n}^{i}=I_{\left\{X_{n}^{i} \geq v_{N-n}^{i}\right\}}(n=1, \cdots, N ; i=1, \cdots, p)$.

Then ${ }^{*} d$ is an equilibrium point for $\operatorname{PROB}(X \mid \pi, N)$ and $v_{N}^{i}=E\left(X_{t_{\pi}\left({ }^{*} d\right)}\right)$. 
We note here that the equilibrium stopping strategy ${ }^{*} d \in D^{i}(X)$ of player $i$ for $\operatorname{PROB}(X \mid \pi, N)$ is determined by $X \in \mathscr{X}^{p \times N}$. Therefore the equilibrium stopping strategy ${ }^{*} d \in D^{i}(X)$ is recognizable or is reflected over $X \in \mathscr{X}^{p \times N}$. Such an individual stopping function will be defined by

$$
{ }^{*} \delta^{i}(X)=\left({ }^{*} \delta_{1}^{i}(X), \cdots,{ }^{*} \delta_{N}^{i}(X)\right)
$$

explicitly expressing of random variable $X \in \mathscr{X}^{p \times N}$.

Theorem 4.1 For a sequence of fuzzy perception functions $\widetilde{X}=\left(\widetilde{X}_{1}, \cdots, \widetilde{X}_{N}\right)$ and a monotone rule $\pi$, a strategy function ${ }^{*} \delta^{i}$ defined in (4.4) gives an equilibrium point for $F-P R O B\{\widetilde{X} \mid \pi, N\}$.

Proof. Let select any $\delta^{i} \in \Delta^{i}$. For $x \in \mathbb{R}$ with $\widetilde{v}^{i}\left({ }^{*} \delta^{-i} / \delta^{i}\right)(x)>0$ and $\varepsilon>0$, there exists $X \in \mathscr{X}^{p \times N}$ such that $E\left(X_{t_{\pi}\left({ }^{*} \delta^{-i} / \delta^{i}\right)}\right)=x$ and $\widetilde{v}^{i}\left({ }^{*} \delta^{-i} / \delta^{i}\right)(x)<\mu_{\widetilde{X}}(X)+\varepsilon$ from the definition of (3.1). Since ${ }^{*} \delta(X)$ is an equilibrium point for $\operatorname{PROB}\{X \mid \pi, N\}$, it follows that $x \leq y \triangleq$ $E\left(X_{t_{\pi}\left({ }^{*} \delta(X)\right)}\right)$ implies $\widetilde{v}^{i}\left({ }^{*} \delta^{i}\right) \geq \mu_{\widetilde{X}}(X)$. And then $\widetilde{v}^{i}\left({ }^{*} \delta^{-i} / \delta^{i}\right)(x)<\widetilde{v}^{i}\left({ }^{*} \delta\right)(y)+\varepsilon$.

Similarly, for any $y \in \mathbb{R}$ with $\widetilde{v}^{i}\left({ }^{*} \delta\right)(y)>0$ and $\varepsilon>0$, we have $\widetilde{v}^{i}\left({ }^{*} \delta^{-i} / \delta^{i}\right)(x)+\varepsilon>$ $\left.\widetilde{v}^{i}{ }^{*} \delta\right)(x)$ provided that $y \geq x \triangleq E\left(X_{\left.t^{(*} \delta(X)\right)}^{i}\right)$. Thus, combining two order relations of (3.3), we have $\widetilde{v}^{i}\left({ }^{*} \delta^{-i} / \delta^{i}\right) \preccurlyeq \widetilde{v}^{i}\left({ }^{*} \delta\right)$.

The next lemma is to prepare an explicit form of the perception value corresponding the equilibrium point. This is a general case for the monotone rule.

Lemma 4.2 For $v=\left(v^{1}, \cdots, v^{p}\right) \in R^{p}$ and $Y=\left(Y^{1}, \cdots, Y^{p}\right) \in \mathscr{X}^{p}$, let, for each $j=1, \cdots, p$,

$$
G^{i}(Y, v) \triangleq v^{i}+\alpha^{i}(v \mid Y) E\left[Y^{i}-v^{i}\right]^{+}-\beta^{i}(v \mid Y) E\left[Y^{i}-v^{i}\right]^{-},
$$

where

$$
\begin{aligned}
& \alpha^{i}(v \mid Y) \triangleq P\left[\pi\left(I_{C^{1}}, \cdots, I_{C^{i-1}}, 1, I_{C^{i+1}}, \cdots, I_{C^{p}}\right)=1\right] \\
& \beta^{i}(v \mid Y) \triangleq P\left[\pi\left(I_{C^{1}}, \cdots, I_{C^{i-1}}, 0, I_{C^{i+1}}, \cdots, I_{C^{p}}\right)=1\right]
\end{aligned}
$$

and $C^{j} \triangleq\left\{Y^{j} \geq v^{j}\right\}$. Then, $G^{i}(Y, v)(i=1, \cdots, p)$ is continuous in $(Y, v) \in \mathscr{X}^{p} \times \mathbb{R}^{p}$.

Proof. Since the underlying $P$ is assumed to be non-atomic by (3.1), the dominated convergence theorem implies that $\alpha^{i}(v \mid Y), \beta^{i}(v \mid Y)$ are continuous in $(Y, v)$. And both of $E\left[Y^{i}-v^{i}\right]^{+}$and $E\left[Y^{i}-v^{i}\right]^{-}$are also continuous. Hence the assertion is obtained immediately.

For a given sequence $\widetilde{X}=\left(\widetilde{X}_{1}, \cdots, \widetilde{X}_{N}\right)$, let define

$$
\widetilde{X}(n) \triangleq\left(\widetilde{X}_{N-n+1}, \widetilde{X}_{N-n+2}, \cdots, \widetilde{X}_{N}\right)
$$

for $n=1, \cdots, N$. By considering the F-PROB $\{\widetilde{X}(n) \mid \pi, n\}$, we denote $\widetilde{v}_{n}=\left(\widetilde{v}_{n}^{i} ; i=1, \cdots, p\right)$ the fuzzy perception value of its equilibrium point.

Theorem 4.2 It holds that, for $n=1, \cdots, N, i=1, \cdots, p$,

(i) $\widetilde{v}_{n}^{i} \in \widetilde{\mathbb{R}}$. 
(ii) The $\alpha$-cut is given as $\widetilde{v}_{n, \alpha}^{i}=\left[-\widetilde{v}_{n, \alpha}^{i},{ }^{+} \widetilde{v}_{n, \alpha}^{i}\right]$ where

$$
-\widetilde{v}_{n, \alpha}^{i}=E\left[-\widetilde{X}_{n, \alpha}^{i}\right], \quad+\widetilde{v}_{n, \alpha}^{i}=E\left[{ }^{+} \widetilde{X}_{n, \alpha}^{i}\right] .
$$

(iii)

$$
\left\{\begin{aligned}
-\widetilde{v}_{n, \alpha}^{i} & =\min _{Y, v} G^{i}(Y, v) \\
+\widetilde{v}_{n, \alpha}^{i} & =\max _{Y, v} G^{i}(Y, v)
\end{aligned}\right.
$$

where the $\min _{Y, v}$ and $\max _{Y, v}$ are taken over the set $\left\{(Y, v) \in \mathscr{X}^{p} \times\left.\mathbb{R}^{p}\right|^{-} \widetilde{X}_{N-n} \leq Y \leq\right.$ $\left.{ }^{+} \widetilde{X}_{N-n}-\widetilde{v}_{n} \leq v \leq+\widetilde{v}_{n}\right\}$ with the order relation $\leq$ of component-wise in $\mathbb{R}^{p}$.

Proof. The proof proceeds by induction on $n$. From the definition of $\alpha$-cut and the expectation of fuzzy set,

$$
\begin{aligned}
\widetilde{v}_{1, \alpha}^{i} & \left.=\left\{x \in \mathbb{R} \mid \widetilde{v}_{1}^{i}{ }^{*} \delta\right)(x) \geq \alpha\right\} \\
& =\left\{E\left(X_{N}^{i}\right) \in \mathbb{R} \mid X_{N}^{i} \in \widetilde{X}_{N, \alpha}^{i}\right\} \\
& =\left[E\left(-\widetilde{X}_{N, \alpha}^{i}\right), E\left({ }^{+} \widetilde{X}_{N, \alpha}^{i}\right)\right],
\end{aligned}
$$

which implies that $\widetilde{v}_{1}^{i} \in \widetilde{\mathbb{R}}$ and (4.8) holds for $n=1$. To prove it for $n$, suppose that (i) and (ii) are hold in case of $n-1$. So

$$
\widetilde{v}_{n+1, \alpha}^{i}=\left\{v_{n+1}^{i}\{X\} \mid \mu_{\widetilde{X}}(X) \geq \alpha, X=\left(X_{1}, \cdots, X_{N}\right) \in \mathscr{X}^{p \times N}\right\}
$$

where $\widetilde{v}_{n+1, \alpha}^{i}$ is given in (4.1) with respect to $X \in \mathscr{X}^{p \times N}$ and $\mu_{\widetilde{X}}(X)$ is in (3.2). Thus, by (4.1), (4.2) and (4.3), we can obtain that

$$
\widetilde{v}_{n+1, \alpha}^{i}=\left\{G_{i}(Y, v) \mid Y \in \widetilde{X}_{N-n, \alpha}(X), v \in \widetilde{v}_{n, \alpha}\right\}=\left[-\widetilde{v}_{n+1, \alpha},{ }^{+} \widetilde{v}_{n+1, \alpha}\right]
$$

holds. This completes the proof of theorem.

\section{An example of a simple majority rule}

In this section, we discuss the case of $p=2$ and the monotone rule is $\pi\left(x^{1}, x^{2}\right)=\max \left\{x^{1}, x^{2}\right\}$, that is, it is a simple majority rule. Applying this case to Theorem 4.2, the sequence of iteration:

$$
{ }^{-} \widetilde{v}_{1, \alpha}^{i}=E\left[{ }^{-} \widetilde{X}_{N, \alpha}^{i}\right], \quad{ }^{+} \widetilde{v}_{1, \alpha}^{i}=E\left[{ }^{+} \widetilde{X}_{N, \alpha}^{i}\right] .
$$

and the recursive equation of (4.9), for $i=1,2$ and $n=1, \cdots, N$,

$$
\left\{\begin{array}{c}
{ }^{+} v_{n+1}^{i}={ }^{+} v_{n}^{i}+E\left({ }^{+} X_{N-n}^{i}-{ }^{-} v^{i}\right)^{+}-P\left({ }^{-} X_{N-n}^{3-i} \geq{ }^{+} v^{3-i}\right) E\left({ }^{+} X_{N-n}^{i}-{ }^{-} v^{i}\right)^{-} \\
{ }^{-} v_{n+1}^{i}={ }^{-} v_{n}^{i}+E\left({ }^{-} X_{N-n}^{i}-{ }^{+} v^{i}\right)^{+}-P\left({ }^{+} X_{N-n}^{3-i} \geq{ }^{-} v^{3-i}\right) E\left({ }^{-} X_{N-n}^{i}-{ }^{+} v^{i}\right)^{-}
\end{array}\right.
$$

are obtained. Thus the fuzzy perception value of equilibrium point is $\left(\widetilde{v}^{1}\left({ }^{*} \delta\right), \widetilde{v}^{2}\left({ }^{*} \delta\right)\right)=\left(\widetilde{v}_{N}^{1}, \widetilde{v}_{N}^{2}\right)$ for an individual stopping strategy function ${ }^{*} \delta=\left({ }^{*} \delta^{1},{ }^{*} \delta^{2}\right)$ with a usual result of fuzzy max order.

To show a concrete numerical calculation considered in Kurano et al. (2004), it is treated an independent, uniformly fuzzy random variables. Let $\widetilde{X}=\left(\widetilde{X}_{1}, \cdots, \widetilde{X}_{N}\right)$ with two players $\widetilde{X}_{n}=\left(\widetilde{X}_{n}^{1}, \widetilde{X}_{n}^{2}\right), n=1, \cdots, N$ and a triangle-fuzzy random variable

$$
\tilde{X}_{n}^{i}=\left(Y_{n}^{i} / X_{n}^{i} / Z_{n}^{i}\right) \quad i=1,2
$$


Table 1: Triangular fuzzy number

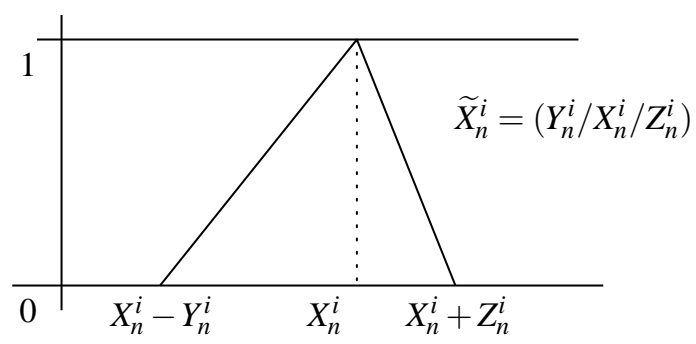

i.e. the center is $X_{n}^{i}$ with a left and a right spread $X_{n}^{i}-Y_{n}^{i}, X_{n}^{i}+Z_{n}^{i}$ respectively. In oder to simplify the calculation, we assume that these are independent and uniformly distributed as

$$
X_{n}^{i} \sim U[0,1], \quad Y_{n}^{i}, Z_{n}^{i} \sim U[0,1 / 2] .
$$

In this case the fuzzy perception value of the equilibrium point $\widetilde{v}_{n}=\left(\widetilde{v}_{n}^{1}, \widetilde{v}_{n}^{2}\right)$ could be explicitly expressed as follows.

Here we use a notation $\mathbb{T}$ and $\mathbb{F}$, a distribution function of $W$, cooperating with a parameter $a$ by

$$
\mathbb{T}(x) \triangleq E(W-x)^{+} \quad(-\infty<x<+\infty)
$$

where random variables $W=X+Y, Z=X-Y$ with $X \sim U[0,1]$ and $Y \sim U[0, a](0<a<1 / 2)$, then

$$
\begin{aligned}
& \mathbb{T}(x)=\left\{\begin{array}{l}
1 / 2+a / a-x,(x<0) \\
1 / 2+a / 2-x+x^{3} /(6 a),(0 \leq x<a) \\
1 / 2+a / 2+a^{2} / 6-(1+a / 2) x+x^{2} / 2,(a \leq x<1) \\
(1+a-x)^{3} /(6 a),(1 \leq x<1+a) \\
0,(1+a \leq x)
\end{array}\right. \\
& \mathbb{F}(x) \triangleq P(W \leq x)=\left\{\begin{array}{l}
0,(x<0) \\
x^{2} /(2 a),(0 \leq x<a) \\
-a / 2+x,(a \leq x<1) \\
-(a+1 / a) / 2-(1+1 / a) x-x^{2} /(2 a),(1 \leq x<1+a) \\
1,(1+a \leq x)
\end{array}\right.
\end{aligned}
$$

in cases like this. Since $E(W-x)^{-}=\mathbb{T}(x)-a / 2-1 / 2+x, E(Z-x)^{+}=\mathbb{T}(x+a), E(Z-x)^{-}=$ $\mathbb{T}(a+x)+a / 2-1 / 2+x$ and $P(Z \leq x)=\mathbb{F}(a+x)$ are hold, we can express the above recursive iteration (5.1) and (5.2) as follows: By the symmetry of players, $\widetilde{v}_{n}^{1}=\widetilde{v}_{n}^{2}$ and, set

$$
\widetilde{u}_{n} \triangleq \widetilde{v}_{n}^{1}=\widetilde{v}_{n}^{2}
$$

and we want to grasp a form of $\alpha$-cut $\widetilde{u}_{n, \alpha}=\left[{ }^{-} \widetilde{u}_{n, \alpha},{ }^{+} \widetilde{u}_{n, \alpha}\right]$ for each $\alpha \in[0,1]$. By substituting $a=(1-\alpha) / 2$,

$$
-\widetilde{u}_{1, \alpha}=1 / 2-(1-\alpha) / 4, \quad{ }^{+} \widetilde{u}_{1, \alpha}=1 / 2+(1-\alpha) / 4,
$$

for $n=1, \cdots, N-1$, we had calculated (5.2) as

$$
\left\{\begin{aligned}
{ }^{-} \widetilde{u}_{n+1, \alpha}={ }^{-} \widetilde{u}_{n, \alpha}+\mathbb{T}( & \left(\widetilde{u}_{n, \alpha}+(1-\alpha) / 2\right)-\left(1-\mathbb{F}\left({ }^{+} \widetilde{u}_{n, \alpha}\right)\right) \\
& \times\left\{\mathbb{T}\left({ }^{+} \widetilde{u}_{n, \alpha}+(1-\alpha) / 2\right)+(1-\alpha) / 4-1 / 2+{ }^{+} \widetilde{u}_{n, \alpha}\right\}, \\
{ }^{+} \widetilde{u}_{n+1, \alpha}={ }^{+} \widetilde{u}_{n, \alpha}+ & \mathbb{T}\left({ }^{-} \widetilde{u}_{n, \alpha}+(1-\alpha) / 2\right)-\left(1-\mathbb{F}\left({ }^{-} \widetilde{u}_{n, \alpha}\right)\right) \\
& \times\left\{\mathbb{T}\left({ }^{-} \widetilde{u}_{n, \alpha}+(1-\alpha) / 2\right)-(1-\alpha) / 4-1 / 2+{ }^{-} \widetilde{u}_{n, \alpha}\right\} .
\end{aligned}\right.
$$


Table 2: The $\alpha$-cuts of the fuzzy equilibrium point

\begin{tabular}{r|c|cc|cc|}
\cline { 2 - 7 } \multicolumn{1}{c|}{} & \begin{tabular}{c}
$\alpha=1$ \\
\multicolumn{1}{c|}{}
\end{tabular} & \multicolumn{2}{c|}{$\alpha=0.7$} & \multicolumn{2}{c|}{$\alpha=0.5$} \\
\hline \hline$n$ & $u[i]$ & lower & upper & lower & upper \\
\hline \hline 1 & 0.5000 & 0.4250 & 0.5750 & 0.3750 & 0.6250 \\
2 & 0.5625 & 0.3811 & 0.7654 & 0.2669 & 0.9004 \\
3 & 0.5890 & 0.2851 & 0.9995 & 0.1494 & 1.2709 \\
4 & 0.6022 & 0.2418 & 1.3124 & 0.1494 & 1.7487 \\
5 & 0.6092 & 0.2418 & 1.6604 & 0.1494 & 2.2265 \\
6 & 0.6130 & 0.2418 & 2.0085 & 0.1494 & 2.7043 \\
7 & 0.6152 & 0.2418 & 2.3566 & 0.1494 & 3.1821 \\
8 & 0.6164 & 0.2418 & 2.7047 & 0.1494 & 3.6599 \\
9 & 0.6171 & 0.2418 & 3.0528 & 0.1494 & 4.1377 \\
10 & 0.6175 & 0.2418 & 3.4008 & 0.1494 & 4.6155 \\
\hline
\end{tabular}

The numerical result is as follows: We use the notation $u[1]=1 / 2, u[n+1]:=1 / 2+$ $u[n]^{3 / 2}, n=1,2, \cdots$ with $a=(1-\alpha) / 2$. The case of $\alpha=1, a=(1-\alpha) / 2=0.0$ corresponds the non-fuzzy model PROB model with the equilibrium value $u[n]$ and, "lower" "upper" means the extremal point of interval for each level $\alpha$.

\section{Acknowledgement}

Authors should show thanks to anonymous referee who point out the typographical errors.

\section{References}

Blackwell, D. (1962). Discrete dynamic programming, Ann. Math. Statist., 33, (1962), 719 726.

Baswell, S. B. and Taylor, M. S. (1987). A central limit theorem for fuzzy random variables, Fuzzy Sets and Systems, 24, 331-344.

Dubois, D. and Prade, H. (1980). Fuzzy Sets and Systems : Theory and Applications, Academic Press.

Fishburn, P. C. (1971). The theory of representative majority decision, Econometrica, 39 , 273284.

Ferguson, T. (2004). Selection by Committee, in Advances in Dynamic Games, A.S. Nowak and K. Szajowski eds., Birkhäuser, 183-188.

Kurano, M., Yasuda, M. Nakagami, J. and Yoshida, Y. (2004). A fuzzy stopping problem with the concept of perception, Fuzzy Optimization and Decision Making, 3, 367-374.

Kurano, M., Yasuda, M. Nakagami, J. and Yoshida, Y. (2005a). Perceptive evalution for optimal discounted rewards in Markov decision processes, Proceeding of MDAI2005 Tsukuba, $25-27$. 
Kurano, M., Yasuda, M. Nakagami, J. and Yoshida, Y. (2005b). Fuzzy optimality equation for perceptive MDPs, Proceeding of IFSA2005 Beijing, 1811-1815.

Nakagami, J., Kurano, M. and Yasuda, M. (2000). A game variant of the stopping problem on jump processes with a monotone rule, Advances in Dynamic Games and Applications (Kanagawa, 1996), Ann. Internat. Soc. Dynam. Games, 5, Birkhäuser, Boston, MA, 257-266.

Puri, M. L. and Ralescu, D. A. (1986). Fuzzy random variables, J.Math. Anal. and Appl., 114, 402-422.

Ramik, J. and Rimanek, J. (1985). Inequality relation between fuzzy numbers and its use in fuzzy optimization, Fuzzy Sets and Systems, 16, 123-138.

Vorobe'v, N. N. (1977). Game Theory, Springer Verlag, New York.

Yasuda, M., Kurano, M., Nakagami, J. (1982). Multi-variate stopping problems with a monotone rule, J. Oper. Res. Japan, 25, 334-349.

Zadeh, L. A. (1965). Fuzzy Sets, Information and Control, 8, 338-353.

Zadeh, L. A. (2002). Toward a perception-based theory of probabilistic reasoning with imprecise probabilities, J. of Statistical Planning and Inference, 105, 233-264. 